\title{
EXPLOITATION OF AM-POTENTIALS BY LINKING MANUFACTURING PROCESSES TO FUNCTION-DRIVEN PRODUCT DESIGN
}

\author{
Reichwein, Jannik (1); Kaspar, Jerome (2); Vielhaber, Michael (2); Kirchner, Eckhard (1) \\ 1: Technische Universität Darmstadt, Product Development and Machine Elements, Germany; 2: \\ Saarland University, Institute of Engineering Design, Germany
}

\begin{abstract}
Additive Manufacturing (AM) processes had an extensively and substantially technological growth over the past years that directly influences the continuously increased and manifold possibilities for processing new and innovative products. However, additively manufactured products mostly are still fabricated with only small adaptions compared to conventional parts, and thus waste many design potentials although specific design guidelines have been widely developed to restrict geometrical deficiencies or suggest improvements in component design.

As a result, this contribution furtherly aims to systematically consider AM potentials already on the functional level of product development offering significant but until now still not or just insufficiently exploited potentials. Therefore, the presented approach uses the already proven Design Pattern Matrix (DPM) approach for conventional technologies extended by a concurrent selection of materials and processes specifically for AM. Here, the DPM derives information about the manufacturing process in form of design elements and links them to the function carriers of the product including a methodological determination of requirements.
\end{abstract}

Keywords: Additive Manufacturing, Design methods, Design for Additive Manufacturing (DfAM), Design Pattern Matrix (DPM)

\section{Contact:}

Reichwein, Jannik

Technische Universität Darmstadt

Produktentwicklung und Maschinenelemente

Germany

reichwein@pmd.tu-darmstadt.de

Cite this article: Reichwein, J., Kaspar, J., Vielhaber, M., Kirchner, E. (2019) 'Exploitation of AM-Potentials by Linking Manufacturing Processes to Function-Driven Product Design', in Proceedings of the 22nd International Conference on Engineering Design (ICED19), Delft, The Netherlands, 5-8 August 2019. DOI:10.1017/dsi.2019.78 


\section{INTRODUCTION}

Owing to increasing demands for individualization, rising share of digitalization and need for shorter time to market, the Additive Manufacturing (AM) of products is becoming an important competitive factor for companies. Therefore, the market for AM is growing and expected to grow further in the next years and decades (Gebhardt, 2016). In doing so, this technology has been developed from solely prototyping to the manufacturing of functional and/or partly structural products (Gibson, 2015). An important step achieving this issue is not only attributable to improvements in processing, but also to extensive progress on an adapted product design. This is because the design of additively fabricated products has to be differentiated to products manufactured with conventional, especially subtractive technologies. Thus, process and material-specific catalogues and design guidelines including special insights on key criteria (e.g., minimum wall thickness, surface qualities, and positioning) were created to support design engineers conceiving producible product parts (Adam and Zimmer, 2014; Kranz, 2017). However, this is just the first step to create high-quality products with additive manufacturing. Moreover, the next step is to use the full constructive potential of the manufacturing technology, i.e. to rethink the whole product function potentially causing a completely beneficial redesign. Consequently, and in contrast to existing approaches for the design for additive manufacturing (DfAM), this contribution presents an approach supporting the synthesis of new ideas instead of merely restricting existing design proposals already within the early product development process, especially taking manufacturing-induced design elements and materials into account.

\section{DESIGN PROCESS AND ADDITIVE MANUFACTURING}

As mentioned before, AM offers multifaceted potentials to improve existing and to make up new products. In order to support the design engineer with potential product improvements, there are several approaches being summarised in the terminology Design for X (DfX). Here, especially Design for Manufacturing (DfM) gains in importance over the years and nowadays is well known and understood. However, these procedures usually provide design guidelines that restrict the geometry of the products in a way that they are producible, but there is a lack of generating new designs instead of restricting existing ones. One of them is the Design Pattern Matrix (DPM) to systematically integrate design elements, which are typical for a manufacturing technology within the design of the product. Using these proposed design elements is beneficial to fulfil the product functions and the manufacturability of components (Roos, 2018).

Consequently, and to get a better comprehension of the following approach, the authors' understanding of the product development process is explained first. According to Pahl et al. (2007), thus the development process is divided into four steps: clarification of the task, conceptual design, embodiment design, and detail design. Passing these four phases, the design engineer specifies its product step-by-step from an initial vision or an idea for improving an existing product up to a detailed product design. During this specification, different product models are used, which are described inside the visualized pyramid, see Figure 1. At each level of the product model pyramid, an iterative process takes place that consists of a further detailing step to generate new ideas and finally evaluating and selecting alternatives.

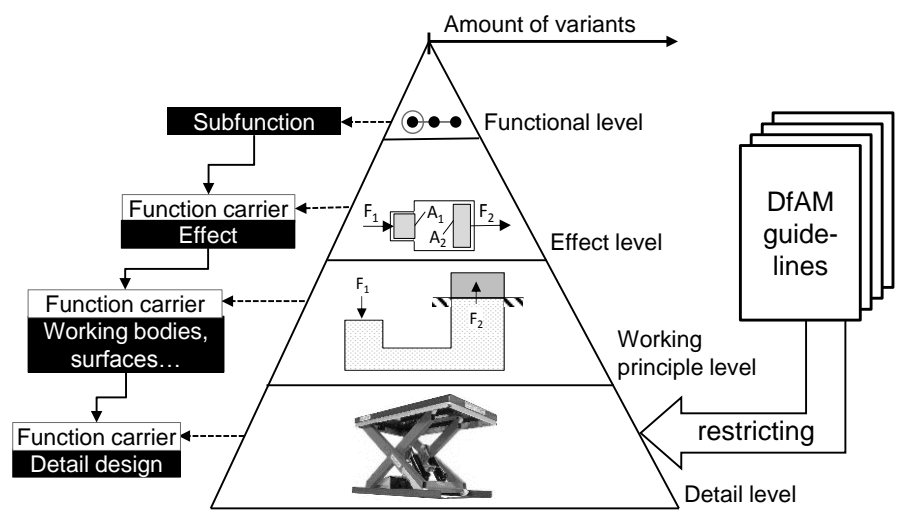

Figure 1. Pyramid of the product models with DfAM guidelines restricting the geometry (adapted from Ehrlenspiel and Meerkamm, 2013) 


\subsection{Current approaches in DfAM}

Having a look to current DfAM approaches, a wide range of the contributions mostly deal with design rules or guidelines that possess geometrical characteristics or limitations of individual AM technologies or even specific materials to prevent the part is not producible (e.g., Adam and Zimmer (2014), Gibson et al. (2015) and Kranz et al. (2015)). Here, and arranged in a logically subdivided order of aspects such as general surface quality depending on orientation, wall thickness or powder removal strategies, the tabular structure facilitates a visualized comparison of exemplary unfavourable and favourable designing solutions added by a more detailed explanatory description. But apart from these basically restrictive DfAM approaches (mainly in terms of AM design catalogues), additionally there are more opportunistic procedures adapting to a situation without paying attention to the limited consequences and own values. One of these are featured by potential analyses assessing, for example, the technological capability to improve lightweight design (possible weight reduction), functions (by functional integration) or development as well as production cost and time aspects especially for small lot sizes with tailored variants (Schmidt, 2016). Furthermore, emerging matters dealing with the implementation of creativity methods such as Design Thinking inside the DfAM process or storing knowledge in digital, tool-based databases (e.g., Senvol ML). Combined solutions looking at a more holistic methodology in theory are recently presented by Kranz (2017) and Kumke (2018). Initial realisations regarding a topology optimization having the general novelty of geometric freedom on the one hand, but still considering the few technical restrictions on the other hand, are also achieved stepby-step (Leary et al., 2016; Robbins et al., 2016; Takezawa and Kobashi, 2017).

However, current approaches still have some lacks when looking at an unbiased analysis at functional design level in order to view on a necessitated complete redesign for AM targeting an overall best product and taking into account all three dimensions of product design, material and process selection. In doing so, design rules mostly in terms of a restrictive manner can just be seen as a basis to integrate the full potentials of AM in an early conceptual phase (see Figure 2). Thus, supporting the designer to handle the constructive potentials of AM at an early stage along with a proper material and manufacturing-induced processing selection based on specific requirements (e.g., strength, media resistances, and tolerances), the successive approach serves as an opportunistic DfAM approach on functional design level.

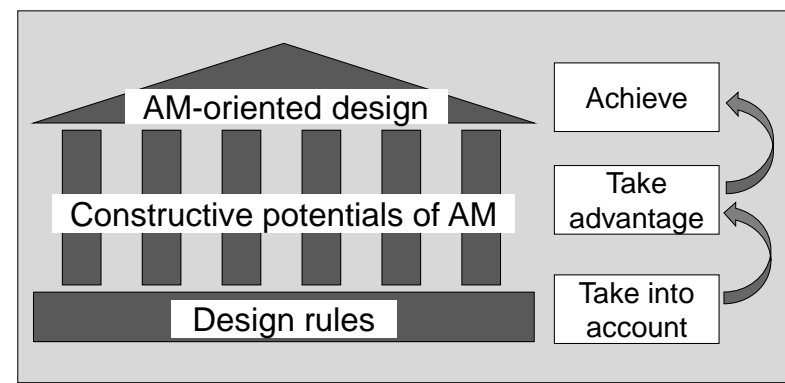

Figure 2. Classification of the approach based on common methods

\subsection{Design Pattern Matrix with manufacturing-induced design elements}

To systematically integrate manufacturing-induced design elements in product development, the Design Pattern Matrix represents a great approach enabling the design engineer to systematically expand the solution space by using information also from the AM point of view (see Figure 3). In order to achieve this, the first step is to formalize the potentials of additive manufacturing in a generalized way to transfer them to different design tasks and products by means of so-called manufacturing-induced design elements. The second step pursues to abstract the product in form of function carriers. These function carriers consist of all elements that are necessary for realising a subfunction and can be applied at the different levels of concretisation of the product model. Next, the linkage of individual manufacturing-induced design elements with respective function carriers is aimed to find useful combinations realising the function carriers' subfunction.

As a result, subsolutions are formed for every subfunction that utilize as much potential of the individual AM technology as possible. Consequently, the outcome of the Design Pattern Matrix is similar to the morphological chart method showing several subsolutions (working principles) for each function of the product. Finally, the last step combines the subsolutions to overall solutions (working 
combinations) and rate them according to the requirements to establish the product's best principlesolution.

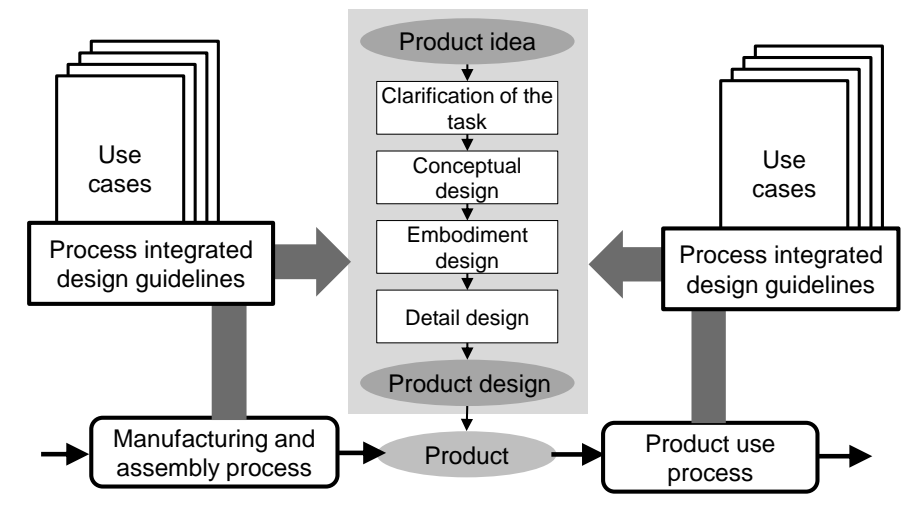

Figure 3. Approach for using process integrated design guidelines originating from manufacturing and use processes during the development (adapted from Roos, 2018)

\section{LINKAGE OF PRODUCT POTENTIALS WITH AM}

Based on the aforementioned approach of the Design Pattern Matrix, it is necessary to store and prepare knowledge about the AM processes and the product use. This knowledge is described in form of process integrated design guidelines to make the potentials of AM available during the design process. Consequently, the next paragraphs analyses the potentials of AM and successful applications.

\subsection{Potentials of additive manufacturing}

Additive manufacturing offers a number of potentials compared to other conventional manufacturing processes. These manifold potentials include, amongst others, new possibilities in the manufacturing of complex geometries and using special materials as well as economic advantages (e.g., in producing individually tailored parts with small lot sizes). Further technical advantages in the fields of material, geometry and product architecture are introduced in the following paragraphs.

\subsubsection{Material diversity}

AM allows to use a broad range of materials. Depending on the particular AM process the use of metals, metal blends and various plastics (e.g., rubbery-elastic materials) is possible. Moreover, materials that are difficult to machine such as titanium, magnesium and ceramics can be processed with less effort than with conventional manufacturing processes. Some of the AM processes enable to manufacture components with gradually or discretely changing a material in one process. Additionally, incorporating inlays of different materials or components is possible. That allows to adapt the stiffness to the load a component has to withstand locally by varying the material's stiffness throughout the component. Thus, the reduction of a component's weight is possible and lightweight design can be performed (Thompson et al., 2016). Likewise, the special behaviour of composites are usable, for example, the bimetal effect of materials with different thermal expansion or purposefully placed anisotropy.

The possibility to use several materials in one process also allows the application of surface coatings during one production step as well as selective colouring for the marking and identification of parts. Different colour pigments as well as different pigment densities can be used in the materials and also the usage of colour changing materials or materials doped with luminescent materials is conceivable (Thompson et al., 2016).

\subsubsection{Geometrical capability}

Geometrical freedoms arise due to the possibility to manufacture complexly bent and roundish shapes without significant additional effort and even inner structures. Subsequently, additive manufacturing enables to manufacture cavities and undercuts. Those geometrical elements are just partly or not at all visible by exposed edges. In conventional manufacturing processes, producing cavities and undercuts is not possible or involves considerable effort. The inner geometries can be bent holes, for example, fluid sewers or cable conduits and also arbitrary and asymmetrical cavities. Using the possibility to 
incorporate arbitrary cavities and undercuts also a certain surface roughness or a defined porosity can be achieved by adjusting the process parameters of the printing process. A proportion between $1 \%$ and $40 \%$ pores is practically feasible whereby the limits strongly depend on the AM processes and their materials. Generating a certain porosity, for instance, is used in membranes, filters and the medicine technology, where the porous structure helps the human's tissue to grow onto the implant. In addition, functional surfaces can be created to reduce the components flow resistance through a surface structure similar to a shark skin.

AM offers further freedoms in shaping like very small filigree structures, which allow to define stiffness and density of any component. Freeform shapes likewise include parts with locally varying wall thickness and shell design. Spherical or curved surfaces and objects insignificantly increase the production effort with additive manufacturing while the effort increases considerably using conventional manufacturing processes. All this entirely leads to structure-optimised parts (e.g., by topology optimisation) that can be manufactured without significant simplifications of the geometry (near-net-shape).

\subsubsection{Product architecture}

In addition to the potentials that arise for individual components, AM offers great potentials when considering the complete assembly. Due to the complex geometry that is possible, several components of an assembly can be combined into one if they are made of the same material and no relative movement between them is necessary. It is possible to integrate additional functions into existing components (e.g., cavities for fluid sewers, cable conduits, and connecting elements). Furthermore, several parts (e.g., ball joints, bearings, and planetary gears) can already be fabricated in an assembled state. Labels with embossed or recessed scripture are produced without additional costs or effort in the same manufacturing step (Gebhardt, 2016). Beyond that, there are some additive manufacturing processes that allow embedding components such as sensors and actuators.

\subsection{Manufacturing-induced design elements}

This approach aims to use the described potentials of the AM technologies during the design process. Therefore, these have to be formalised first in order to enable the design engineer to use them in any kind of realisation. The formalised description bases on an abstract level in form of manufacturinginduced design elements. The manufacturing-induced design elements are derived from the analysis of a large number of successfully fabricated products with AM, a knowledge-based database.

On geometric level, there are eight different design elements. For example the third design element derived from the geometric freedom of the additive manufacturing technology is the ability to produce undercuts. While it is a challenge to produce a part shown in Figure 4 with conventional manufacturing technologies, it is mostly not with AM. A visualised part like this with various undercuts is no more difficult to fabricate than a geometry without undercuts.

Another typical design element for AM is a three-dimensional truss structure. This kind of structure often is the result of lightweight design, for example, from topology optimization or bionic design methods. Apart from the macro-level, this structure is also applied on meso-level (lattice-structures) and micro-level (porous structures) as design elements.

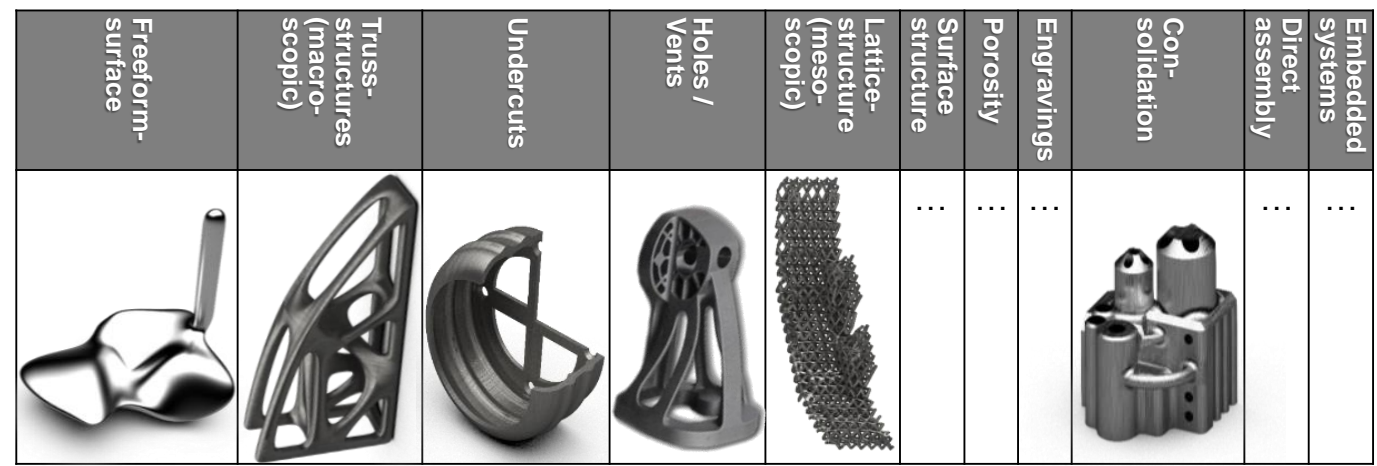

Figure 4. Manufacturing-induced design elements for additive manufacturing 
In addition, AM technologies enable changes in the product architecture, and thus have the potential to reduce the number of parts or to integrate new functions into existing parts which leads to big savings in material use and costs. Consequently, changes in product architecture have to be considered during the design process. Here, mainly three design elements were recognized mostly being successfully used in existing constructions (see Figure 4). The first one is the consolidation of parts in terms of adding new functions to an existing part or to combine two or more parts into one joint part. The second design element is to produce already assembled components; and the last is to embed other components in the additively manufactured part such as sensors. A large number of examples and use cases in form of a catalogue exist for all the design elements described which may be used to raise creativity.

\subsection{Methodological product analysis}

Originating from an existing product to adopt AM, the first step is to analyse the product function and structure. To achieve a good design suitable for additive manufacturing, it is important to move away from solely existing solutions, especially if the current product is manufactured with subtractive technologies. Therefore, it is advantageous to abstract the geometry of the product in order to obtain the product functions (see Figure 5). Considering an original design where no predecessor product exists, the analysis can be skipped and the procedure starts with the definition of the product functions.

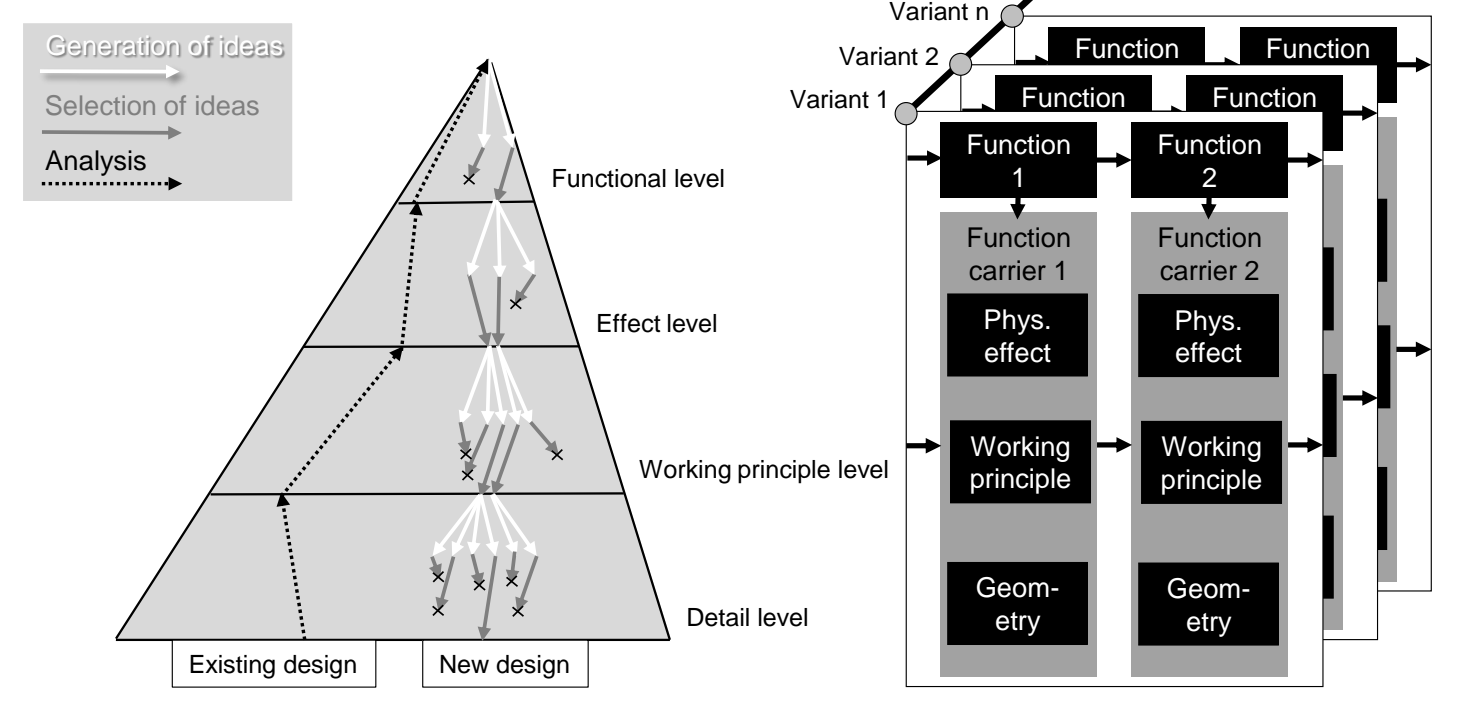

Figure 5. Methodological analysis of existing product

Finally, the different subfunctions of the product are arranged in a function structure and each subfunction leads to a function carrier. These function carriers consist of all elements that are necessary to fulfil the corresponding subfunction and can be defined in different levels of abstraction (effect, working principle and detail level).

\subsection{The AM design pattern matrix}

The linkage of information from manufacturing and product use processes to the product design is a decisive step of this approach. The DPM can be used to link the design elements of additive manufacturing with the function carriers of the product (Würtenberger et al., 2018). Therefore, the DPM is composed of function carriers in the first column and design elements in the headline (see Figure 6). The design engineer can now choose for every cell in the matrix if this design element can be used in the function carrier to fulfil the specific sub-function. In addition, it is possible to use several design elements in combination to build up the function carrier.

This leads to several ideas represented by an exclamation mark in Figure 6, which can be further specified to subsolutions for each function carrier or respective subfunction of the product. The further detailing of the initial ideas is symbolized through a highlighted pencil. If there is more than one idea per line, they can be combined to one subsolution or it can lead to several working principles for the relevant function carrier. Analogous to the morphological chart method, the subsolutions then will be assembled to overall solutions (working combinations). In the further progress of the product 
development process this concepts will be selected and evaluated according to diverse requirements the product has to fulfil. Thus, all principle solutions have in common that the potential of AM was systematically and, at the same time, integrally taken into during the initial product development process.

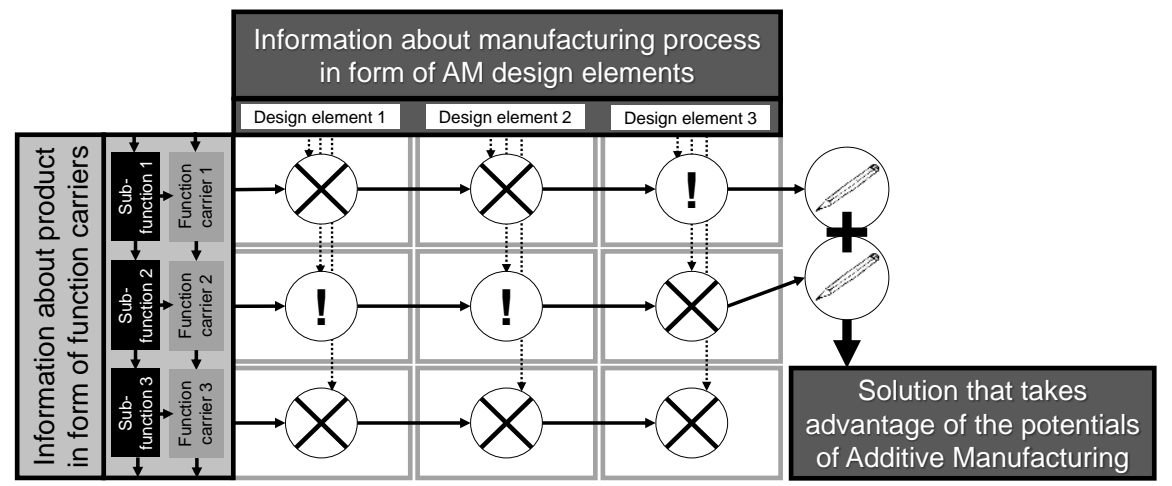

Figure 6. Design pattern matrix for the linkage of design elements and function carriers

\section{CONCURRENT SELECTION OF MATERIAL AND PROCESS}

Based on the aforementioned linkage of manufacturing-induced design elements and function carriers, a next step within a fully integrated view is additionally represented by a more detailed discussion on requirements for materials and manufacturing processes. Thus, a concurrent selection targeting a specification of appropriate technological options and alternatives leads to a viable geometric design through the Design Pattern Matrix (see Figure 7).

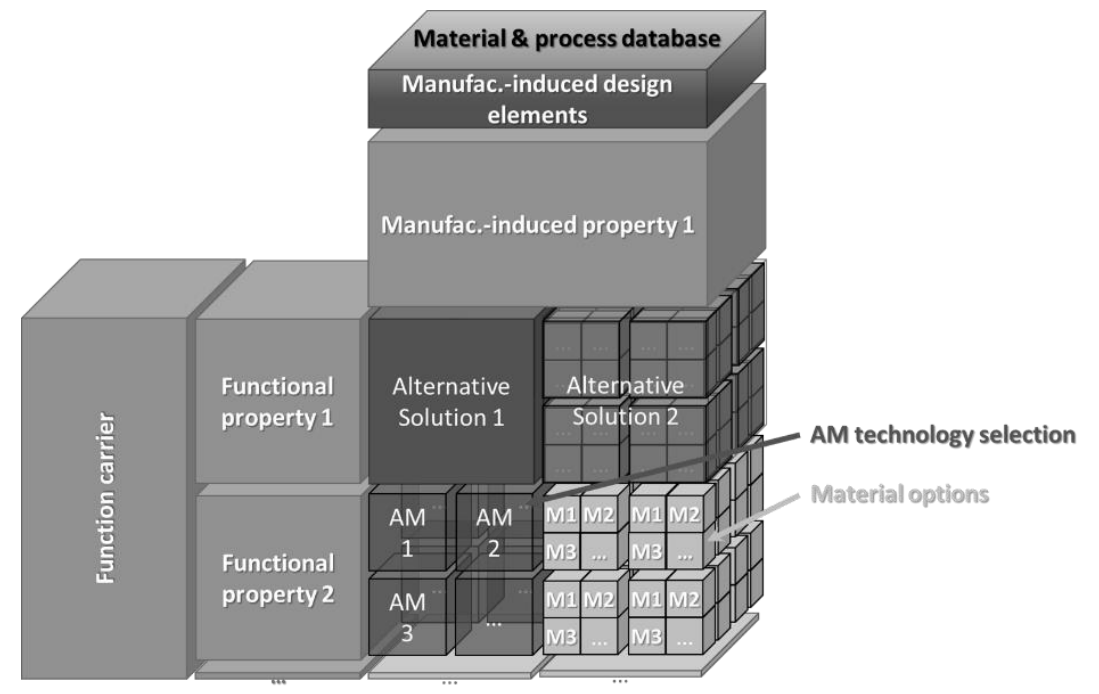

Figure 7. Significance of concurrent selection of material and process in DPM

Here, first a gathering of material and processing-relevant criteria is addressed in correlation with the initially proposed working principles. If this is done for all principle solution variants out of a knowledgebased data pool with respect to every function carrier, the screening of individually remaining materials to fulfil these diverse requirements (e.g., strength, density/weight, and resistance to a multiplicity of media) is next, analogous to Ashby's approach (Ashby, 2011). In doing so, a more accurate determination of not only functionally proposed but rather technologically (material and manufacturing) feasible solution variants is guaranteed. This means, an initial design proposal that meets the principle function could be still sorted out by material properties which necessitates high strength requirements, and thus just permits an adjusted metal-based AM realisation despite strict cost requirements; or technologically calls for very precise surface patterns in the viewable area and accurate dimensional tolerances resulting in stereolithographic processing techniques (without recognising a separately downstream post-processing step) along with their range of available materials but is still impracticable due to limitations in the demanded building size. Figure 8 serves as visualised explanation of the appreciation process. 


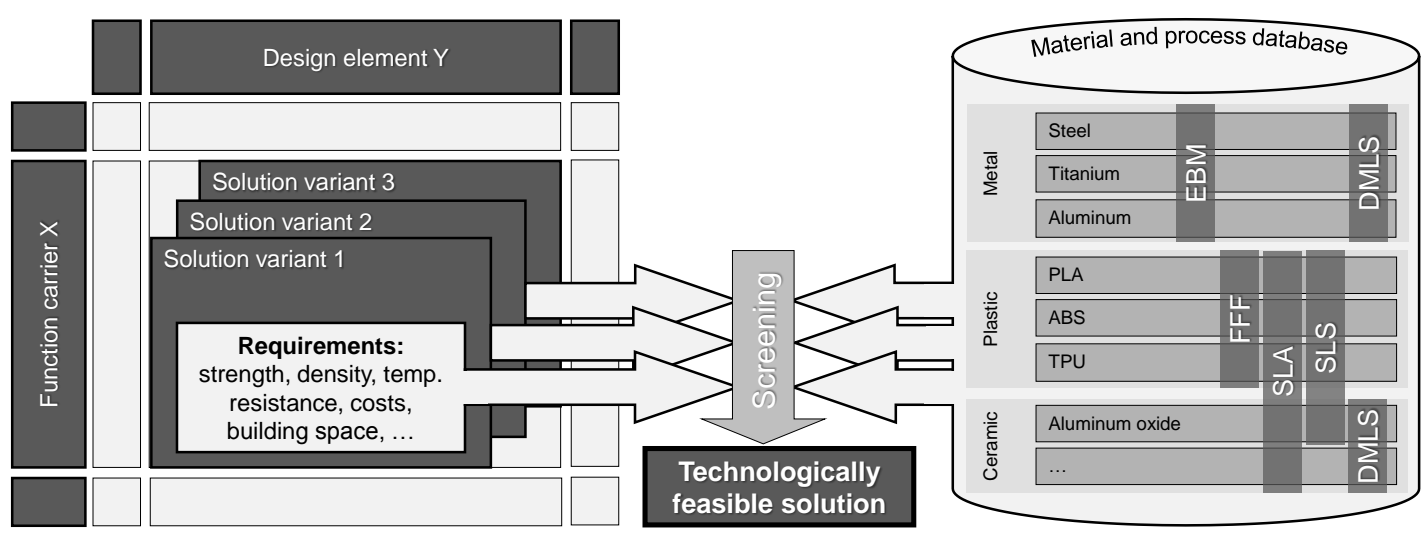

Figure 8. Concurrent screening of materials and processes for DPM solutions alternatives

However, and coming up with remaining and well-proven alternative solution pairs (individually function carrier based solution variants of working principle, choice of material and process definition), the investigation of a most desirable and efficient concept of a particular function carrier depends on a sufficient assessment. Consequently, a two-step evaluation process takes place. First, the left materials and processes get separately not just assessed on a fulfilled / not-fulfilled basis (binding criteria) like it is done in the initial screening process. Rather, they are subject to an overall weighted technical, economic and ecological sufficiency ranking (Kaspar et al., 2018) displaying a percentage performance level based on local and global but solution-neutral requirements. Second, and in combination with the ultimately proposed alternative solutions, the material and processing options are embedded with any design of the respective working principle and correlatively valued once more in a further integrated view. As a result, finally the best solution variants with a technological feasibility turned out.

\section{APPLICATION EXAMPLE}

In literature there are several approaches for selecting part candidates for additive manufacturing, e.g. Lindemann et al. (2015). These can be used in a first step to choose promising parts out of a company's portfolio. Based on that, the presented approach can be used for a potential analysis of a complete redesign of selected parts in order to gain all the benefits provided by AM technologies.

Thus, the evaluation of the approach is applied to the adaptation design of an injection-moulding tool. At first, it is necessary to analyse the existing product. The function of an injection-moulding tool is to produce parts with a defined geometry from liquefied thermoplastic polymers. The tool provides a geometric shape that is the negative of the latter product. This shape is filled with liquefied plastic and then cooled down to cure the material. After this, the finished part is ejected out of the tool. As a result, the subfunctions of this system are filling of the form, providing shape, cooling down, and ejecting the part out of the tool.

This leads to the function structure shown in Figure 9, where a function carrier is derived for each subfunction. Here, the function carriers are described on working principle level represented by sketches. Thus, the function structure and the function carrier form the first two rows of the Design Pattern Matrix opposite to the manufacturing-induced design elements developed in section 3.2 on the top of the matrix. The combination of design elements and function carriers in each cell leads to new concepts that are using the potentials of AM. Figure 9 shows a few exemplary concepts for the injection-moulding tool generated through the application of the Design Pattern Matrix. For the function "provide shape", for example, the design engineer can use truss and lattice structures to reduce the mass of the tool. Usually weight is not an important factor for an injection-moulding tool, but in this case it is more cost-efficient to produce a lattice structure than solid material. In contrast to subtractive manufacturing, where any further removal of material is more expensive, thus additive manufacturing leads to less material usage and shorter production time.

In addition to the geometric design elements, a concurrent selection of material and manufacturing processes takes place through the specification of requirements for each function carrier. The material of the tool has to withstand temperatures up to $250^{\circ} \mathrm{C}$ to hold the liquid plastic and, at the same time, has to offer dimensional stability. This criterion already excludes few materials and also manufacturing processes, for example, there is no common plastic that can fulfil these requirements. 


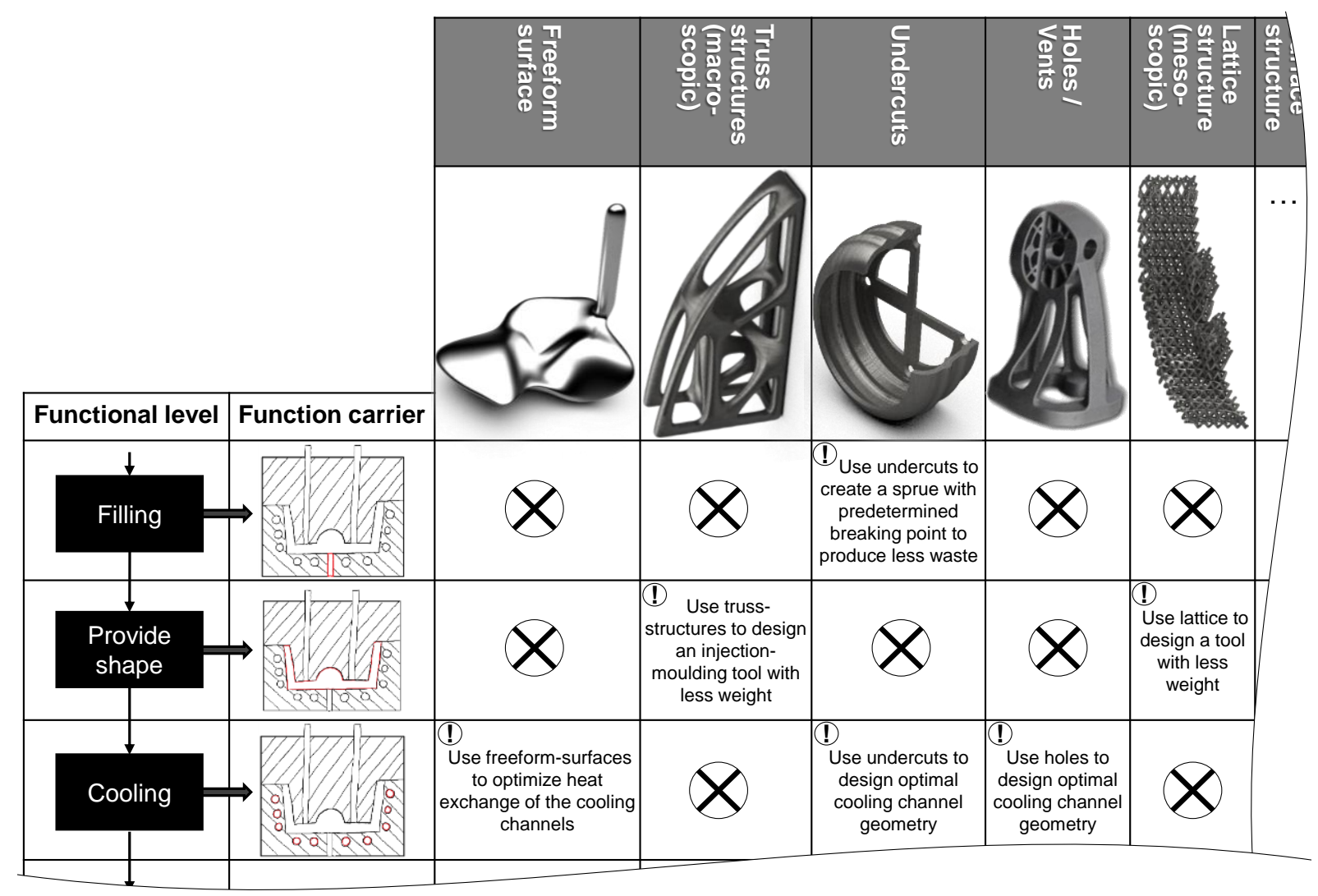

Figure 9. Application of design pattern matrix for injection-moulding tool

\section{CONCLUSION}

The presented approach uses information from manufacturing and product use processes to systematically extend the solution space during the early phases in product development. These additional solutions make particular use of the advantages of AM processes to fulfil the functions of the product. Accordingly, this approach is an appropriate addition to the already existing restrictive design guidelines, and moreover allows inexperienced designers to develop products with an AMoriented design. In the further procedure, the individual subsolutions for the function carriers must be combined and evaluated to form overall solutions. The concepts, for example, can be specified at the working principle level and must therefore still be completed at the detail level. For products that are manufactured additively anyway, numerical optimisations are a good option to specify the geometry, especially to achieve lightweight design but also to reduce material usage. During this specification of the embodiment design, the design engineer can use the proven design guidelines to obtain a technologically feasible product.

Having discussed a concurrent selection of materials and processes within the presented Design Pattern Matrix for additive manufacturing, this procedure could be enhanced further on to crossfunctional aspects according to a generally more systemic vision of Kaspar and Vielhaber (2016), i.e. in terms of investigations for integral designs throughout fundamentally same material and processing properties for a number of different function carriers within the entire product system (Kaspar et al., 2019).

Conclusively, further work can be done in the area of constructing the concepts from the Design Pattern Matrix to their final design on embodiment level. Therefore, numerical optimisation and specific design proposals directly inside diverse CAD software tools can be used. Additional research will be done on the specification of the single design elements (e.g., how to design lattice structure), the assembling of sub-concepts in the DPM to overall concepts, and the systematically assessment of solutions to find the overall best solution. 


\section{REFERENCES}

Adam, G.A.O. and Zimmer, D. (2014), "Design for Additive Manufacturing - Element transitions and aggregated structures”, CIRP Journal of Manufacturing Science and Technology, Vol. 7 No. 1, pp. $20-28$. https://doi.org/10.1016/j.cirpj.2013.10.001

Ashby, M.F. (2011), Materials selection in mechanical design, Butterworth-Heinemann, Oxford. https://doi.org/10.1016/C2009-0-25539-5

Ehrlenspiel, K., Meerkamm, H. (2013), Integrierte Produktentwicklung. Denkabläufe, Methodeneinsatz, Zusammenarbeit, Hanser, München

Gebhardt, A. (2016), Additive Fertigungsverfahren. Additive Manufacturing und 3D-Drucken für Prototyping Tooling - Produktion, Hanser, München.

Gibson, I., Rosen, D., and Stucker, B. (2015), Additive Manufacturing Technologies: 3D Printing, Rapid Prototyping, and Direct Digital Manufacturing, Springer-Verlag, New York. https://doi.org/10.1007/9781-4939-2113-3

Kaspar, J. and Vielhaber, M. (2016), "Cross-Component Systematic Approach for Lightweight and MaterialOriented Design”, DS 85-1: Proceedings of NordDesign 2016, Vol. 1, pp. 332-341.

Kaspar, J., Choudry, S.A., and Vielhaber, M. (2018), "Concurrent Selection of Material and Joining Technology - Holistically Relevant Aspects and Its Mutual Interrelations in Lightweight Engineering”, Procedia CIRP, Vol. 72, pp. 780-785. https://doi.org/10.1016/j.procir.2018.03.093

Kaspar, J., Reichwein, J., Kirchner, E., and Vielhaber, M. (2019), "Integrated Design Pattern Matrix for Additive Manufacturing - A Holistic Potential Analysis for Systemic Product and Production Engineering", Procedia CIRP, Vol. XX, p. XXX-XXX. (To be published)

Kranz, J., Herzog, D., and Emmelmann, C. (2015), "Design guidelines for laser additive manufacturing of lightweight structures in TiAl6V4", Journal of Laser Applications, Vol. 27 No. S1, pp. 14001.1-14001.16. https://doi.org/10.2351/1.4885235

Kranz, J. (2017), Methodik und Richtlinien für die Konstruktion von laseradditiv gefertigten Leichtbaustrukturen, Springer-Verlag, Berlin Heidelberg. https://doi.org/10.1007/978-3-662-55339-8

Kumke, M. (2018), Methodisches Konstruieren von additiv gefertigten Bauteilen, Springer, Berlin Heidelberg. https://doi.org/10.1007/978-3-658-22209-3

Leary, M., Merli, L., Torti, F., Mazur, M., and Brandt, M. (2014), “Optimal topology for additive manufacture: A method for enabling additive manufacture of support-free optimal structures", Materials and Design, Vol. 63, pp. 678-690. https://doi.org/10.1016/j.matdes.2014.06.015

Lindemann, C., Reiher, T., Jahnke, U., Koch, R. (2015), "Towards a sustainable and economic selection of part candidates for additive manufacturing”, Rapid Prototyping Journal, Vol. 21 No. 2, pp. 216-227. https://doi.org/10.1108/RPJ-12-2014-0179

Pahl, G., Beitz, W., Feldhusen, J., Grote, K.-H. (2007), Konstruktionslehre-Grundlagen erfolgreicher Produktentwicklung; Methoden und Anwendung, Springer, Heidelberg. https://doi.org/10.1007/978-3-54034061-4

Robbins, J., Owen, S.J., Clark, B.W., and Voth, T.E. (2016), “An efficient and scalable approach for generating topologically optimized cellular structures for additive manufacturing", Additive Manufacturing, Vol. 12 No. B, p. 296-304. https://doi.org/10.1016/j.addma.2016.06.013

Roos, M. (2018), Ein Betrag zur einheitlichen Modellierung und durchgängigen Nutzung fertigungstechnologischen Wissens im Produktentwicklungsprozess. Dissertation Technische Universität Darmstadt.

Schmidt, T. (2016), Potentialbewertung generativer Fertigungsverfahren für Leichtbauteile, Springer-Verlag, Berlin Heidelberg. https://doi.org/10.1007/978-3-662-52996-6

Takezawa, A. and Kobashi, M. (2016), "Design methodology for porous composites with tunable thermal expansion produced by multi-material topology optimization and additive manufacturing”, Composites Part B: Engineering, Vol. 131, p. 21-29. https://doi.org/10.1016/j.compositesb.2017.07.054

Thompson, M.K., Moroni, G., Vaneker, T., Fadel, G., Campbell, R. I., Gibson, I., Bernard, A., Schulz, J., Graf, P., Ahuja, B., Martina, F. (2016), "Design for Additive Manufacturing”, CIRP Annals, Vol. 65, No. 2. 2016, pp. 737-760.

Würtenberger, J., Reichwein, J., and Kirchner, E. (2018), "Using the potentials of additive manufacturing by a systematic linkage of the manufacturing process to product design", proceedings of the DESIGN 2018 15th International Design Conference, pp. 1465-1576. https://doi.org/10.21278/idc.2018.0225 